Recent Insights into the Physics of the Sun and Heliosphere:

Highlights from SOHO and Other Space Missions

IAU Symposium, Vol. 203, 2001

P. Brekke, B. Fleck, and J. B. Gurman eds.

\title{
Waves in the Magnetised Solar Atmosphere
}

\author{
C. S. Rosenthal, M. Carlsson, V. Hansteen, A. McMurry \\ Institute of Theoretical Astrophysics, University of Oslo, Box 1029, \\ N-0315, Blindern, Oslo, Norway \\ T. J. Bogdan, S. McIntosh
}

HAO/NCAR, Box 3000, Boulder, Colorado, CO 80307-3000, USA

\author{
Å. Nordlund \\ NBIfAFG, Københavns Universitet, Juliane Maries Vej 30, DK-2100 \\ København Ø, Denmark \\ R. F. Stein \\ Dept. of Physics and Astronomy, Michigan State University, East \\ Lansing, MI 48824, USA
}

S. B. F. Dorch

The Royal Swedish Academy of Sciences, Stockholm Observatory, SE-133 36 Saltsjöbaden, Sweden

\begin{abstract}
We describe simulations of the propagation of waves in magnetised structures in the solar atmosphere.
\end{abstract}

\section{Introduction}

Direct measurement of magnetic field strengths and configurations above the solar photosphere is difficult. As an alternative to spectroscopic techniques, we propose the use of MHD waves as probes of the magnetic structure of the solar atmosphere. (For coronal applications see the review by Roberts (2000), and also Nakariakov \& Régnier et al. in these proceedings).

\section{Method And Results}

We solve the equations of magnetohydrodynamics using the numerical approach described by Nordlund \& Galsgaard (1995). We describe here calculations of sinusoidal waves driven through $2 \mathrm{D}$, potential magnetic fields, embedded in an isothermal, non-radiating atmosphere. These restrictions allow us to explore a wide range of problems at relatively low computational expense, with an emphasis on fundamental MHD processes rather than detailed physical simulation. 


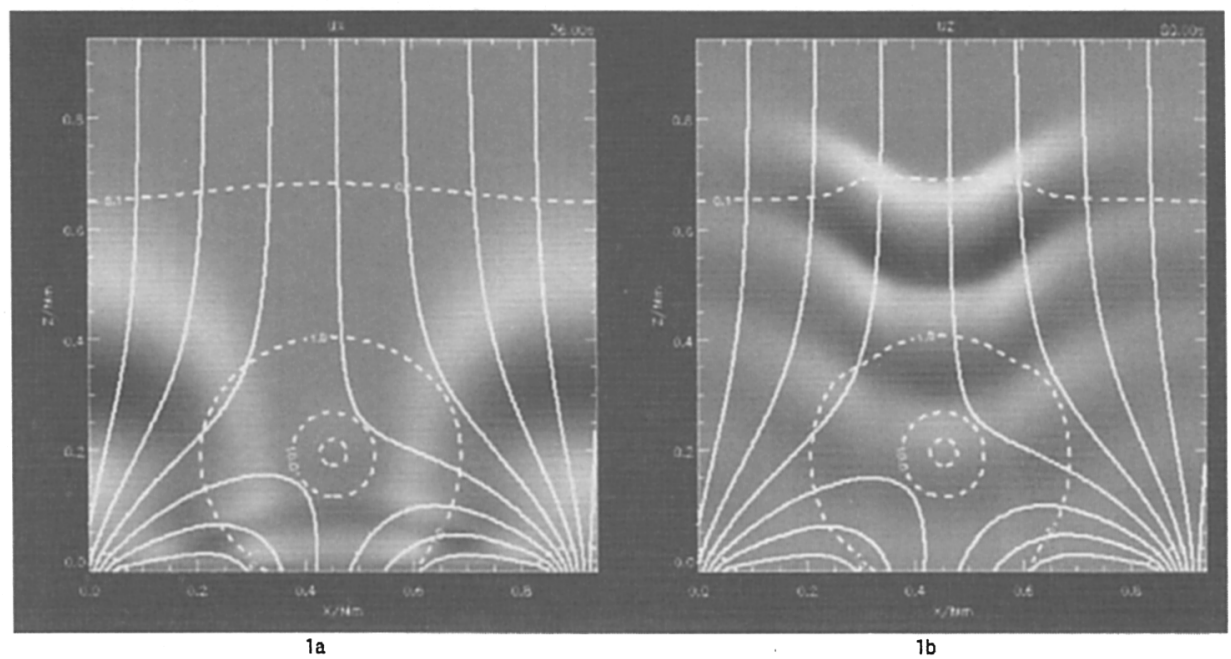

Figure 1. Snapshots from two simulations of wave-propagation in the vicinity of an $\mathrm{x}$-type neutral point. The solid lines are field lines and the dashed lines are contours of plasma- $\beta$. The scale height is $160 \mathrm{~km}$ and the box is $6 \times 6$ scale-heights. The left (right) panel shows the horizontal (vertical) velocity component in a simulation driven with a horizontal (vertical) motion at the lower boundary. The frequency is $42 \mathrm{mHz}$.

Figure 1 shows wave propagation near an $\mathrm{x}$-type neutral point. Shear waves propagate along the field lines (left panel) and so are trapped in closed-field regions but propagate out to infinity along open field lines. The exponential increase of Alfvén speed with height causes the reflection of all non-zero horizontal wavenumbers so that the purely Alfvénic wave which reaches the top of the domain contains no information about the underlying field structure.

In the longitudinally driven case (right panel), the waves propagate everywhere, but at higher speed in regions where they cross the field lines due to the additional restoring force from the magnetic pressure. Wavefronts are therefore focussed away from such regions. Moreover, the steepening of the wave-fronts with height is more rapid in regions where the propagation speed is lower. Hence, at chromospheric heights, upwardly propagating shocks should be less visible in and above regions containing significant horizontal or inclined field.

Figure 2 shows wave propagation in a geometry representing a possible configuration of magnetic field in a sunspot. The field is now more intense and the open-field region, corresponding to the sunspot umbra, is now many scaleheights across. In the penumbral (closed-field) region, the wave propagation speed is very high and the wave front becomes highly deformed, eventually reflecting back downwards, due to the increasing Alfvén speed, and interfering with the upwardly traveling wave-fronts. The result is a series of outwardly propagating wave caustics in the penumbral atmosphere. The umbral dynamics is much simpler, consisting of train of steepening, convex, acoustic (MHD slowmode) wave fronts channeled along the field. 


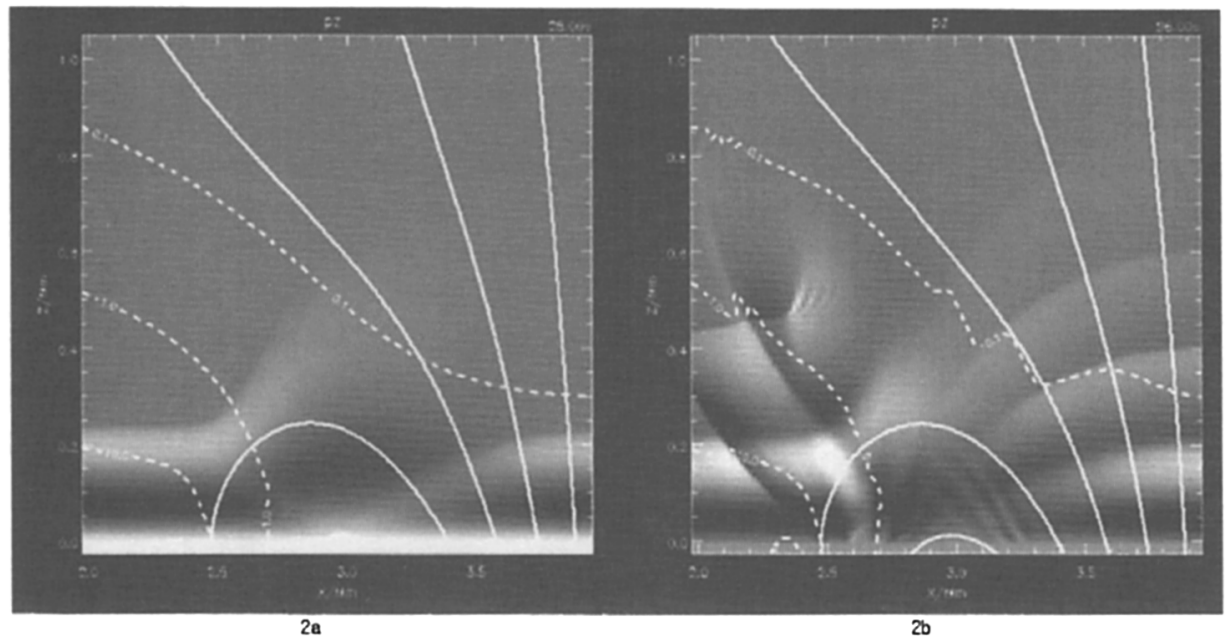

Figure 2. Two snapshots of vertical momentum density from a single simulation of longitudinally driven waves of frequency $42 \mathrm{mHz}$ in a sunspot. Note that the plot uses a false aspect ratio. Only a section of the whole ( $8 \mathrm{Mm}$ wide) simulation is shown.

\section{Conclusions}

Figure 1 suggests how waves in the chromosphere may be used to track the underlying field configuration. We intend to extend this analysis by modeling waves propagating in fields extrapolated from measured surface values and comparing the results with chromospheric observations such as those of Muglach (these proceedings)

The results of the sunspot calculation are strongly suggestive of the curious dichotomy that, at chromospheric heights, waves in umbrae take the form of "umbral flashes" - trains of shock-like brightenings, almost uniform across the umbra, while the penumbra shows "running penumbral waves" - outwardly propagating waves which appear to be dynamically coupled to the umbral flashes (Tsiropoula et al. 2000)

Acknowledgments. This research was funded in part by the EC TMR Programme through the European Solar Magnetometry Network.

\section{References}

Roberts, B. 2000, Sol. Phys., 193, 139

Nordlund $\AA$. \& Galsgaard, K. 1995, http://www.astro.ku.dk/ aake

/NumericalAstro/papers/kg/mhd.ps.gz

Tsiropoula, G., Alissandrakis, C.E., \& Mein, P. 2000, A\&A, 355, 375 